\title{
Quality Assessment of Pansharpened RASAT and Landsat-8 Images Using Synthetic Sentinel-2 Panchromatic Image
}

\author{
Mehmet Akif Günen ${ }^{1,2}$ \\ ${ }^{1}$ Erciyes University, Department of Geomatics Engineering, Faculty of Engineering, Kayseri \\ ${ }^{2}$ Gümüshane University, Department of Geomatics Engineering, Faculty of Engineering and Natural Sciences, Gümüşhane
}

KEY WORDS: Pansharpening, Sentinel-2, RASAT, Landsat-8, NIQE, BRISQUE.

\begin{abstract}
:
Technical and physical limitations often do not allow images to be acquired with high spatial and spectral resolution. Pansharpened images obtained by fusing high spatial resolution panchromatic images and multi-spectral images are widely used in GIS applications. In this study, it is aimed to increase the spatial resolution of the RASAT and Landsat-8 multispectral satellite images with synthetic Sentinel-2 panchromatic data. Six different pansharpening methods were used to test the success of the synthetic panchromatic data generation method using dataset with two different land use/land cover properties. Seven full reference image quality assessment metrics and two referenceless image quality assessment metrics were used to perform quantitative comparison.
\end{abstract}

\section{INTRODUCTION}

There is an increase in the use of optical satellite imagery in geographic information system applications (Zhang and Mishra, 2012), defense industry (Fang et al., 2013), urban area planning (El-Mezouar et al., 2012), precision agriculture applications (Jenerowicz and Woroszkiewicz, 2016), forestry (Rahmani et al., 2010) and mining application (Yokoya et al., 2016). The technical and physical features of satellite sensors, in which optical satellite images are captured, are unique. Related sensors have the capability to capture data in a certain frequency range. In order to obtain high spectral resolution images, the related frequency range is kept narrow and the number of photons collected in the sensor is reduced. Thus, the signal to noise ratio decreases with spatial resolution. Optical-based Panchromatic (PAN) images and Multispectral images (MS) simultaneously can be obtain the same spatial area on earth with different or same satellites. PAN images provide high spatial resolution while MS images provide high spectral resolution. Pansharpening is the pixel-based image fusion of MS images with PAN images to obtain high spatial resolution MS images. Pansharpening is a topic that has been studied for many years because high spatial and spectral resolution images cannot be obtained directly from all imaging systems due to technical constraints and financial possibilities. Each satellite produces a remote sensing optical image with a different resolution level. Sensors with high spatial resolution are costly and require a lot of storage space. With the pansharpening methods, the images are improved, the classification accuracy of the related images is increased, and the visual interpretation is strengthened. In the basic context, the pansharpening process can be modeled as removing the relevant sharpness detail information from the PAN image and adding it to the MS image. Various methods have been developed in the literature for the process of pansharpening. Qualitative and quantitative comparisons are usually made to compare the success of the relevant methods. Visual data related to the protection of spectral information is presented with qualitative comparison. As a result of quantitative comparison, changes occurring at the end of the pansharpening process in MS images are analyzed using various metrics (Vivone et al., 2014; Zhang and Mishra, 2012; Garzelli et al., 2004; Günen, 2021).
In this paper, two different satellite image datasets were used for pansharpening. Here, a synthetic PAN was created from the Sentinel-2 image. Landsat- 8 OLI and RASAT optical satellite images were pansharpened with various methods using the related Sentinel-2 PAN image. Thus, spatial resolutions of Landsat- 8 and RASAT images are increased. Although many pansharpening methods have been developed, the most popular such as Smoothed Filter Intensity Modulation (SFIM), HueSaturation-Value (HSV), Intensity-Hue-Saturation (IHS), Principal Component Analysis (PCA), BROVEY and GramSchmidt (GS), were preferred. For the quantitative comparison of the methods; Root Mean Square Error (RMSE), Correlation Coefficient (CC), Spectral Angle Mapper (SAM), Relative Average Spectral Error (RASE), Erreur Relative Globale Adimensionnelle de Synthèse (ERGAS), Structural Similarity Index (SSIM), Peak Signal-to-Noise Ratio (PSNR), Naturalness Image Quality Evaluator (NIQE) (Mittal et al., 2013) and Blind/Referenceless Image Spatial Quality Evaluator (BRISQUE) (Mittal et al., 2011)metrics were used.

\section{MATERIAL}

In this paper two different optical satellite image, RASAT and Landsat-8, was used. RASAT satellite images operated by TÜBITAK Space Technologies Research Institute and can be accessed from gezgin-geoportal (URL, 2020). Here, various levels of data can be downloaded. The RASAT image was launched in 2011 and the visible bands of the RASAT image have a 15 meter spatial resolution and 8-bit radiometric resolution (Teke et al., 2014). The RGB bands of the Landsat- 8 satellite image used in the study have a spatial resolution of 30 meters and a 12-bit radiometric resolution. Sentinel-2 PAN image has a 15 meter spatial resolution and 12-bit radiometric resolution (Korhonen et al., 2017). In order to test the success of the proposed PAN generation method, experiments were carried out in two different datasets. All applications in this paper were made using Matlab and ArcGIS software. The Dataset-1 shown as Area-1 in Figure 1 is Istanbul, Turkey. Images of the relevant area were obtained with RASAT and Sentinel-2 and acquisition dates are given in Table 1 . The Dataset-2 shown as Area-2 in Figure 2 is Elbistan, Kahramanmaraş, Turkey. Images of the relevant area were obtained with Landsat- 8 and Sentinel- 2 and acquisition dates are given in Table 1. 


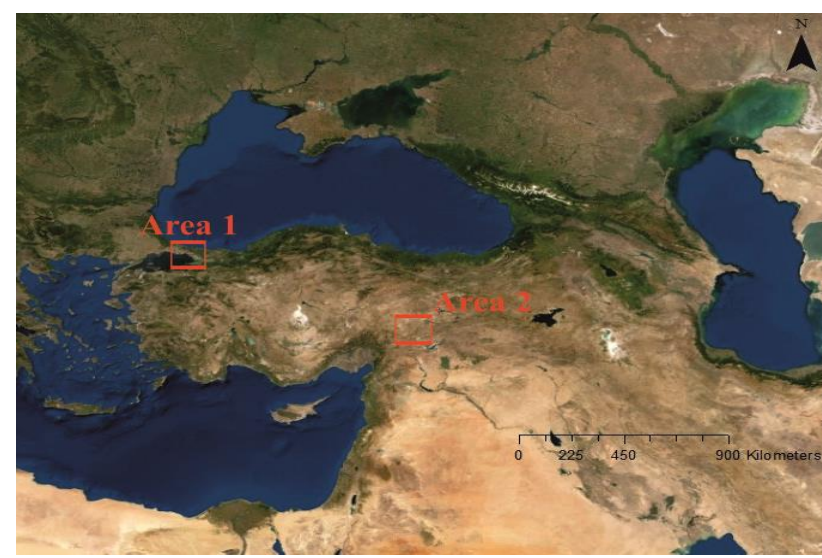

Figure 1. Study Areas on Turkey Satellite Image

Istanbul is the most populous city in Turkey, it has the traffic jams and dense residential area. For this reason, urban areas of Istanbul are constantly being monitored. Area- 2 was designated as Elbistan because it includes road, water, and agricultural land in the narrow region. As it has the fourth largest lowland in Turkey, it has extensive agricultural areas and wetlands. Also, it is a region that is constantly monitored with satellite images because it receives immigration.

\begin{tabular}{|c|c|c|}
\hline \multirow{2}{|c|}{ Data } & Acquisition Date \\
\hline \multirow{2}{*}{ Dataset-1 } & Sentinel 2A & $19 / 11 / 2019$ \\
\cline { 2 - 3 } & RASAT RGB & $18 / 11 / 2019$ \\
\hline \multirow{2}{*}{ Dataset-2 } & Sentinel 2A & $18 / 09 / 2019$ \\
\cline { 2 - 3 } & Landsat 8 OLI & $23 / 09 / 2019$ \\
\hline
\end{tabular}

Table 1. Acquisition date of Sentinel 2A, RASAT and Landsat8 for study areas

\section{METHODS}

In order to increase the spatial resolution of Landsat- 8 and RASAT satellite images, a synthetic PAN image was created using RGB and NIR band of the Sentinel-2 image as in the related reference paper (Borana et al., 2019). However, unlike the related paper, visible bands of Landsat- 8 and RASAT MS images were pansharpened. The methods used for the pansharpening process are introduced in this section. PAN and MS images with different spatial resolution must be expressed at the same spatial resolution in order to apply the related methods. To do this, the MS image must be upsampling (i.e. near or cubic methods) to PAN image resolution. The methods of pansharpening used in this paper are briefly introduced. The data are corrected products containing surface reflection data from the USGS's Level 1 Terrain (L1T) Landsat 8-OLI and the European Space Agency's Level 2A Sentinel 2A.

In SFIM method, a new image is obtained by first dividing the PAN image into itself after the average filter is applied. Then, the spectral values are maintained by dividing each band of the MS image into the corresponding new image. If MS and PAN images are not well georeferenced, there are significant residues in the images (Vivone et al., 2014; Zhang and Mishra, 2012; Vivone et al., 2014). The IHS method often used in remote sensing studies is based on color space conversion. In IHS, the image is converted from the RGB color space to the IHS color space. Assuming that the intensity band corresponds to the panchromatic image, it is replaced by the panchromatic image. A high resolution multi-spectral image is obtained by reverse IHS transformation. The HSV method is quite similar to the IHS method, but the HSV color space is used instead of the IHS color space (Teke et al., 2014; Fang et al., 2013). In the PCA method in which MS image bands are converted to principal components, the color information cannot be preserved too much since the PAN image contains spatial information. In the related method, the first component of the MS image with the highest variance is replaced by the PAN image. The BROVEY method is a process based on modulating the brightness information of the MS image with the PAN image. Here, each band of the MS image is multiplied by the PAN image and the results are divided by the sum of the MS bands (Zhang and Mishra, 2012; Vivone et al., 2014). First of all, the average of the MS image is accepted as a PAN image. Then, backtransform is performed by replacing the high resolution PAN image with the lowest resolution after being decorrelated by the Gram-Schmidt orthogonalization method (Maurer, 2013).

\section{RESULTS}

Qualitative and quantitative comparison methods are generally used to compare pansharpening methods. Because of visual comparison is often insufficient, quantitative comparison should be done. After performing visual comparison, the images with pansharpening process should be compared statistically with the help of some mathematical metrics. In addition to the most frequently used full reference image quality assessment metrics in the literature, a comparison was made with the two methods referenceless image quality assessment. Brief descriptions of the image quality assessment metrics used in this paper are as follows; RMSE is the metric representing the square mean of the difference between the reference image and the pansharpened image. CC is the metric that expresses the correlation value between images. The SAM metric is based on the similarity between the vectors and the success of the related pansharpening method increases as the metric value decreases. RASE is derived from RMSE, success of the metric increases as the numerical value is decreases. ERGAS is the metric that takes into account the spatial resolution ratios of MS and PAN images together with the RMSE metric. In order to determine the image quality, SSIM and PSNR, which are the most frequently used full reference image quality assessment metrics were used. Also, NIQE and BRISQUE, which are referenceless quality assessment metrics, were used. Quantitative evaluation of Dataset 1 results obtained with the related pansharpening methods according to related metrics is given in Table 2 .

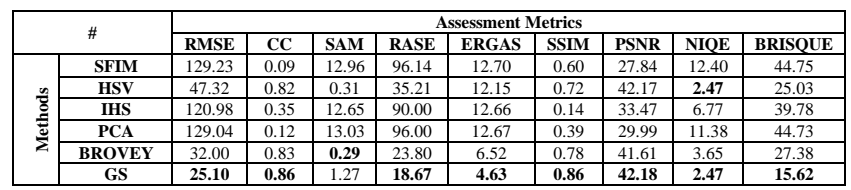

Table 2. Statistical results of Dataset 1. (+Bold express the most successful result.)

When Figure 2 is examined, it is seen that GS method produces a value very close to the MS image given in Figure 2.a. Figure 2 is given to make a qualitative comparison of the related pansharpening methods. While SFIM provided the worst quantitative result, BROVEY provided the most successful result after GS. Quantitative evaluation of Dataset 2 results obtained with the related pansharpening methods according to related metrics is given in Table 3 . When Table 3 is examined, it is seen that GS method produces successful results as in Dataset 1 . Figure 3 is given to make a qualitative comparison of the related methods. Here, SFIM produced the lowest NIQE and BRISQUE values, although it produced high CC values. Although visually SFIM and GS seem to produce close, residues are seen in SFIM method. 


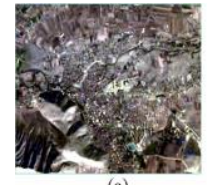

(a)

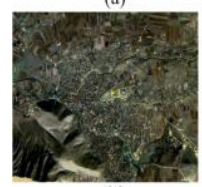

(e)
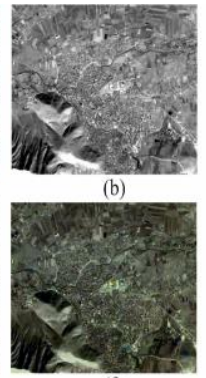

(f)

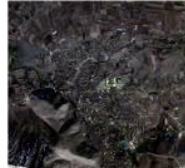

(c)

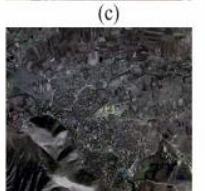

(g)

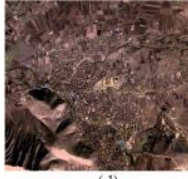

(d)

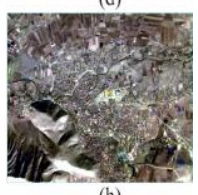

Figure 2. Qualitative results of Landsat-8 dataset a) MS, b) PAN, c) SFIM, d) HSV, e) IHS, f) PCA, g) BROVEY, and h) GS

\begin{tabular}{|c|c|c|c|c|c|c|c|c|c|c|}
\hline \multicolumn{2}{|c|}{$\#$} & \multicolumn{10}{|c|}{ Assessment Metric } \\
\cline { 2 - 11 } & RMSE & CC & SAM & RASE & ERGAS & SSIM & PSNR & NIQE & BRISQUE \\
\hline \multirow{4}{*}{} & SFIM & 14.89 & $\mathbf{0 . 7 8}$ & 0.11 & 13.78 & 3.41 & 0.44 & 36.72 & 7.36 & 36.27 \\
\cline { 2 - 10 } & HSV & 68.61 & 0.62 & $\mathbf{0 . 0 9}$ & 63.49 & 10.72 & 0.18 & 34.70 & 4.50 & 22.97 \\
\cline { 2 - 10 } & IHS & 38.75 & 0.73 & 2.05 & 35.86 & 6.99 & 0.34 & 23.03 & 4.07 & 21.40 \\
\cline { 2 - 10 } & PCA & 54.30 & 0.62 & 0.29 & 50.24 & 24.14 & 0.22 & 31.75 & 4.16 & 22.02 \\
\cline { 2 - 10 } & BROVEY & 76.80 & 0.61 & 0.23 & 71.07 & 11.32 & 0.18 & $\mathbf{2 2 . 6 9}$ & 4.50 & 27.88 \\
\cline { 2 - 10 } & GS & $\mathbf{1 3 . 8 7}$ & 0.67 & 1.34 & $\mathbf{1 2 . 8 4}$ & $\mathbf{3 . 2 3}$ & $\mathbf{0 . 5 7}$ & 37.70 & $\mathbf{3 . 9 0}$ & $\mathbf{1 8 . 2 0}$ \\
\hline
\end{tabular}

Table 3. Statistical Results of Dataset 2. (+Bold express the most successful result.)
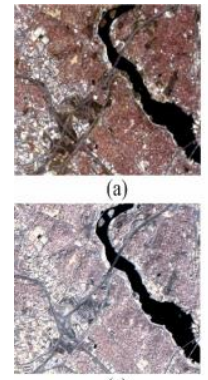

(e)
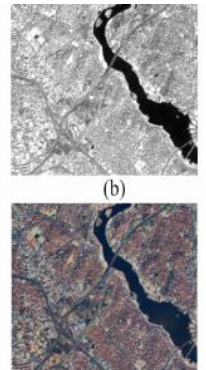

(f)

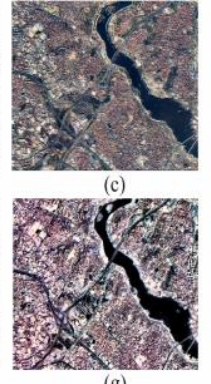

(g)

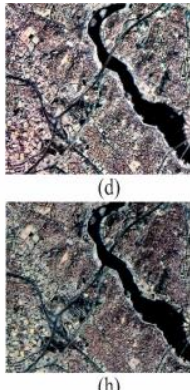

(h)
Figure 3. Qualitative results of Rasat dataset a) MS, b) PAN, c) SFIM, d) HSV, e) IHS, f) PCA, g) BORVEY, and h) GS

When Figure 3 is examined, it is seen that the pansharpened images that are not close to the MS image. Here, the best result was again the GS method. When Figure 2 and Figure 3 are evaluated together, it is seen that SFIM method produces residual results in both. Here, the model is trained by taking training data from the relevant Landsat- 8 and RASAT images to produce image quality assessment metrics with the NIQE and BRISQUE methods. Thus, results were produced from the related methods. Local color quality, in addition to global color quality, must be investigated for a more detailed assessment (Figure 4 and Figure 5). As a result, CCs were calculated between pansharpened images and specific land use/cover areas chosen randomly from the original MS.

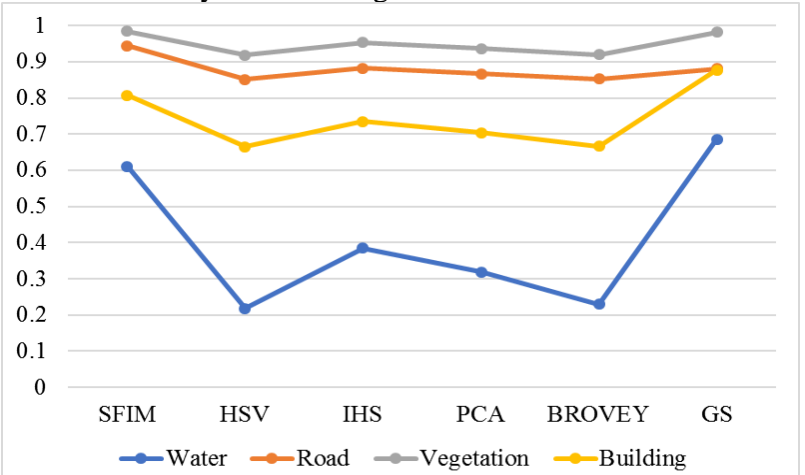

Figure 4. Correlation coefficients calculated for different land use types for Dataset 1

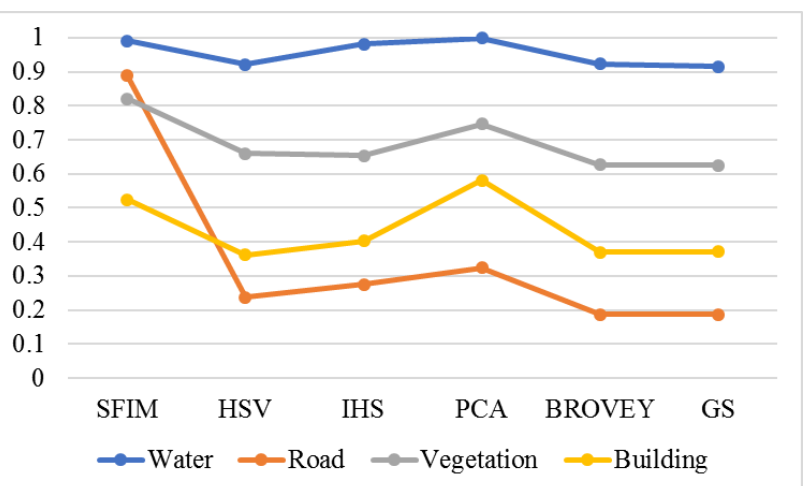

Figure 5. Correlation coefficients calculated for different land use types for Dataset 2

GS method in Dataset 1 SFIM in Dataset 2 was found to be most successful in color preservation of different land use types. In general, high success has been achieved in keeping the colors of vegetation and building classes.

\section{CONCLUSION}

In this paper, Landsat and RASAT images were sharpened by using a synthetic PAN image created using the Sentinel-2 image. Six different methods were used for this process. Satellite images with different spatial resolutions and obtained with different sensors were used. The results are also compared with the referenceless image quality assessment methods (i.e. NIQE and BRISQUE), in addition to the well-known image quality assessment metrics. Qualitative comparison differs little from quantitative research. As a result of all experiments, the GS method was the best method, but SFIM was the most unsuccessful method. In addition, the availability of the proposed PAN generation method on RASAT image, as well as the Landsat 8 image, which is often used in the literature for pansharpening processing, has been determined with statistical tools and visual presentations. It should be noted that RASAT images are presented to users at different levels. The image used in this study is L1, that is, a radiometric corrected image. It can be said that the main reason why the RASAT image has a worse visual presentation than the Landsat- 8 image is the problem experienced in georeferencing. In future studies, the most suitable pansharpening algorithms (i.e. deep learning and traditional based) will be determined by comparing them to improve classification accuracy, distinguish crop patterns, detect mining areas, etc.

\section{REFERENCES}

El-Mezouar, M. C., Taleb N., Kpalma K., Ronsin J., 2012. Edge preservation in ikonos multispectral and panchromatic imagery pan-sharpening. 1st Taibah University International Conference on Computing and Information Technology

Fang, F., Li F.,Shen C., Zhang G., 2013. A variational approach for pan-sharpening. IEEE Transactions on Image Processing 22 (7):2822-2834

Garzelli, A., Nencini F., Alparone L., Aiazzi B., Baronti S., 2004. Pan-sharpening of multispectral images: A critical review and comparison. IEEE International Geoscience and Remote Sensing Symposium 
Günen, M. A., 2021. Weighted differential evolution algorithm based pansharpening. International journal of remote sensing 42 (22):8468-8491

Jenerowicz, A., Woroszkiewicz M., 2016. The pan-sharpening of satellite and UAV imagery for agricultural applications. Remote Sensing for Agriculture, Ecosystems, and Hydrology

Korhonen, L., Packalen P., Rautiainen M., 2017. Comparison of Sentinel-2 and Landsat 8 in the estimation of boreal forest canopy cover and leaf area index. Remote sensing of environment 195:259-274

Maurer, T., 2013. How to pan-sharpen images using the GramSchmidt pan-sharpen method-a recipe. International archives of the photogrammetry, remote sensing and spatial information sciences

Mittal, A., Moorthy A. K., Bovik., A. C., 2011. Blind/referenceless image spatial quality evaluator. Forty fifth asilomar conference on signals, systems and computers

Mittal, A., Soundararajan R., Bovik A. C., 2013. Making a Completely Blind Image Quality Analyzer. IEEE Signal Process Letters 20 (3):209-212

Rahmani, S., Strait M., Merkurjev D., Moeller M., Wittman T., 2010. An adaptive IHS pan-sharpening method. IEEE Geoscience and Remote Sensing Letters 7 (4):746-750

Borana S. L., Yadav S. K., Parihar S.K., Kaplan G. J., 2019. Pan-Sharpening of Landsat-8 Using Synthetic Sentinel-2 PAN Data. Esri India User Conference

Teke, M., Seyfioğlu M. S., Ağçal A., Gürbüz S. Z., 2014. RASAT Uydu Görüntülerinin Optimal Pankeskinleştirilmesi Optimal Pansharpening of RASAT Satellite Imagery, https://www.gezgin.gov.tr/pansharp/RASAT_SIU_2014.pdf

URL, https://gezgin.gov.tr/. Last Acces Date:15/01/2020

Vivone, G., Alparone L., Chanussot J., Dalla M., Garzelli A., Licciardi G. A., Restaino R., Wald L., 2014. A critical comparison among pansharpening algorithms. IEEE Transactions on Geoscience and Remote Sensing 53 (5):25652586

Yokoya, N., Chan J. C.-W., Segl K.. 2016. Potential of resolution-enhanced hyperspectral data for mineral mapping using simulated EnMAP and Sentinel-2 images. Remote Sensing 8 (3): 172

Zhang, Y., Mishra R. K.. 2012. A review and comparison of commercially available pan-sharpening techniques for high resolution satellite image fusion. 2012 IEEE International Geoscience and Remote Sensing Symposium 